\title{
Crystal plasticity extend FEM implementation of thermal-tensile aluminum alloy
}

\author{
Yang Liu', Yiguo Zhu ${ }^{1}$, Liang Ying ${ }^{1}$ and Ping $\mathrm{Hu}^{1, a}$ \\ ${ }^{1}$ State Key Laboratory of Structural Analysis for Industrial Equipment, Faculty of Vehicle Engineering and Mechanics, Dalian University of \\ Technology, 2 Linggong Road, Dalian 116023, China
}

\begin{abstract}
Multi-level approach has been used to simulate the thermal deformation of aluminium alloy at different temperature and strain rate. The crystal plasticity model is extended in the finite element method and the thermal behaviour is integrated in the constitutive equations. Moreover, the damage evolution is also reflected in the simulation using continuum damage mechanics model. Thus, the void evolution and thermal effect could both be shown in the simulation. A new shear strain rate model is constructed with the thermal activated mechanism to describe the rate dependent behaviours during tensile test. The thermal parameters are determined in a fitting test of representative volume element to compare with the experimental data. The results prove that the mechanical tensile behaviour of 5052 aluminium alloy could be well described at different temperatures. The damage evolution process is expressed by the stress concentration and strain concentration in the finite element simulation, which are also confirmed by the experiments.
\end{abstract}

\section{Introduction}

Aluminium alloy has become one of most popular metal in the vehicle industry. The steel is replacing by the aluminium alloy for reducing the weight and protecting the environment. The high specific strength and good resistance allow aluminium alloy to form car components, such as trunk, bumper, and engine hood with good properties. To produce the components using aluminium alloy, the thermal mechanical behaviours should be simulated and controlled in the appropriate range, since the formability of aluminium alloy is small in the room temperature. The traditional phenomenological models lack inner illustration of material mechanism and metallographic essence of microstructure. In the microscopic view, anisotropic plastic behaviour is a primary phenomenon in the crystallography and the dislocation dynamics could be its most significant cause. In addition to the deformation, the crystallographic anisotropy also leads to the different kinds of orientation dependent behaviours, such as strain hardening and void evolution.

In this sense, to better understand the relationship between material property and microstructure of aluminium alloy, various multi-level polycrystalline schemes are built and developed to describe this orientation property such as Taylor and Saches models. These early attempts have already simulated the texture and lattice reorientation well, but the grain interaction and complicated boundary condition could not be explicitly revealed in these homogenization methods.
After the 1980s, a crystal plasticity extended finite element method (CPFEM) occurred in 2D or 3D framework [1-3]. Nowadays, it has become a widely-used method to simulate different kinds of materials and complicated outer boundary conditions. The stress heterogeneity and material behaviours near grain boundaries are betters understood using CPFEM. Each grain scale level of CPFEM is hierarchically constructed by associated constitutive laws accounting for microstructure properties. Green's function fast Fourier transform (FFT) and spectral interpolation is also combined within CPFEM to efficiently and accurately simulate the orientation-dependent mechanism at the grain interface $[4,5]$.

Considering more complex outer and inner effects, such as the temperature, strain rate, recrystallization, and dislocation mobility, the mechanical response could be influenced and deviated enormously from the ordinary condition. Therefore, more complicated model are needed to describe these effects, and the constitutive laws are generally revised to satisfy more complicated cases. As for thermal effects, a visco-plastic self-consistent (VPSC) model is generally used to homogenize the polycrystal and calculate the mechanical behaviour as a function of strain rate and temperature [6]. This advanced mean-field scheme is able to simulate the temperature field coupled with mechanical field, but still lack of capturing codeformation within grains. In view of spatial stress distribution, 3D CPFEM framework is used and the thermodynamically-consistent framework is integrated to characterize the heat conduction and the heat boundary

\footnotetext{
${ }^{\mathrm{a}}$ Corresponding author: pinghu@dlut.edu.cn
} 
condition. Another thermomechanical coupling could calculate the heat generation of deformation recovery, plastic work dissipation and stored cold work motivated by the rearrangement of defects [7, 8].

Here, integrated in the CPFEM simulation, the damage evolution of continuum damage mechanics model and thermal activated mechanism are involved in describing the thermomechanical behaviours and void evolution process of aluminium alloy at elevated temperature. The thermal-associated parameters should be obtained by the fitting test, which could be done by introducing a representative volume element (RVE). Along with the jump test, all the parameters could be confirmed in the RVE simulation and later used in the CPFEM simulation of actual thermal tensile test. The practical specimen of aluminium alloy is simulated and the necking behaviour is also compared to a reduced domain, and localized deformation and void evolution are shown in the 3D large plastic strain simulation. This article is constructed as follows: In chapter 2, the multiscale framework is introduced; in chapter 3, the thermal constitutive equations and the damage functions are briefed; then, the thermal parameters are obtained in the RVE simulation and the jump test is also introduced; in chapter 4, the simulation of actual thermal tensile test is briefed; the conclusion and discussion are brought in chapter 5 .

\section{Framework of Crystal Plasticity Extended Finite Element Method}

This hierarchical model is based on the framework of crystal plasticity and implemented in the finite element method. In the finite element method, the displacement field is calculated by the discretized virtual work principle and it will be checked at each increment. Thus, the stress equilibrium is satisfied at the boundary of grains, moreover, the strain compatibility is also fulfilled among grains in a weak numerical sense.

$$
\boldsymbol{F}=\boldsymbol{F}^{e} \boldsymbol{F}^{p} \boldsymbol{F}^{\ominus}
$$

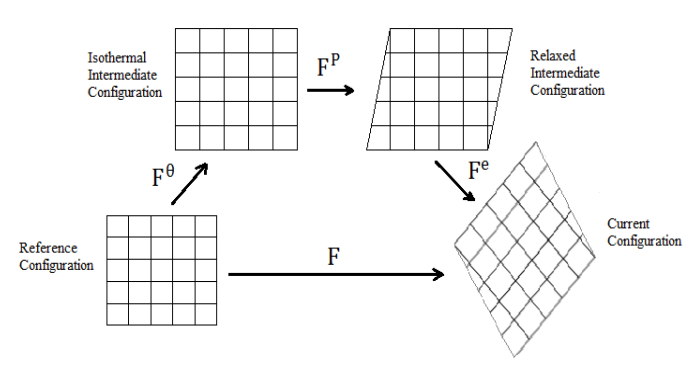

Figure 1. Sketch illustration of the revised configuration evolution and multiplicative decomposition of deformation gradient.

The crystal plasticity model is coupled with thermal effect and damage evolution, which is inserted in the subroutine Use-defined Material Mechanical Behaviour (UMAT) of commercial software ABAQUS. The constitutive model of crystal plasticity method is revised. As for the kinematic relation, the multiplicative decomposition of deformation gradient is modified due to thermal influence.

The original elastic and plastic deformation gradients are $\mathrm{F}^{\varepsilon}$ and $\mathrm{F}^{\mathrm{p}}$, respectively. It should be noticed that $\operatorname{det} \mathrm{F}^{e}>1$ and $\operatorname{det} \mathrm{F}^{p}=1$, which are due to the lattice stretching and the incompressible plastic volume. $\mathrm{F}^{\theta}$ accounts for the isothermal deformation gradient, which is defined as,

$$
\boldsymbol{F}^{\theta}=\left[1+\mu\left(\theta-\theta_{0}\right)\right] \boldsymbol{I}
$$

where, $\theta$ and $\theta_{0}$ are the current and initial absolute temperature, and $\mathbf{I}$ is the unit tensor.

The deformation gradient is related to the subroutine UMAT at the start of each step in ABAQUS. And the basic constitutive equations are used.

$$
\begin{gathered}
\boldsymbol{E}=\frac{1}{2}\left(\boldsymbol{F}^{\boldsymbol{\varepsilon}^{\top} T} \boldsymbol{F}^{\varepsilon}-D\right) \\
\boldsymbol{T}=\boldsymbol{F}^{\varepsilon-1}\left\{\left(\operatorname{det} \boldsymbol{F}^{\varepsilon}\right) \boldsymbol{\sigma}\right\} \boldsymbol{F}^{\varepsilon-T}
\end{gathered}
$$

Other traditional kinematic equations are defined as follows to relate the microscopic crystal structure to the macroscopic mechanical deformation field.

$$
\begin{aligned}
& \boldsymbol{F}^{p}=\boldsymbol{L}^{p} \boldsymbol{F}^{p} \\
& \boldsymbol{L}^{p}=\sum_{\alpha} \dot{\gamma}^{\alpha} \overline{\boldsymbol{P}}_{0}^{\alpha} \\
& \boldsymbol{F}_{\mathrm{t}+\Delta t}^{p} \doteq\left(\boldsymbol{I}+\Delta t \boldsymbol{L}_{t+\Delta t}^{p}\right) \boldsymbol{F}_{\mathrm{t}}^{p}
\end{aligned}
$$

where $\overline{\boldsymbol{P}}_{0}^{\alpha}$ is the initial Schmidt tensor, $\mathbf{L}^{\mathbf{P}}$ the plastic velocity gradient, $\dot{\gamma}^{\alpha}$ the shear strain rate in the $\alpha$ th slip system. Equation (7) is the time incremental representation of updating $\mathrm{F}^{\mathrm{P}}$.

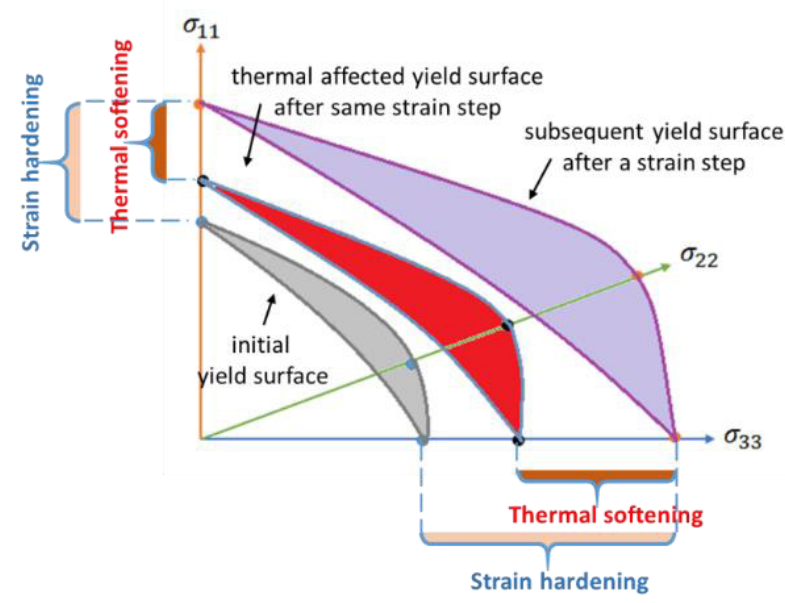

Figure2. The yield surface changing with strain hardening and thermal softening in the stress space.

According to the thermal activated mechanism, the evolution of shear strain rate is revised instead of the power-law equation.

$$
\dot{\gamma}^{\alpha}=\frac{\rho_{m} v_{i d} b^{2}}{2} \exp \left(\frac{-\Delta F}{k \theta}\right) \exp \left(\frac{\left(\tau^{\alpha}-s^{\alpha}\right) \Delta V}{k \theta}\right)
$$


where, $\rho_{m}$ is the mobile dislocation density, $v_{i d}$ is the vibration frequency of this segment of dislocation, $\mathrm{b}$ is the Burger's vector, $v_{\mathrm{g}}$ the average gliding dislocation velocity, $\mathrm{k}$ the Boltzmann constant, $\Delta \mathrm{F}$ and $\Delta \mathrm{V}$ the thermal activated energy and volume.

In addition to the shear strain rate function, the strain hardening rate is also revised.

$$
\begin{aligned}
& \dot{s}^{\alpha}=\sum_{\beta} h^{\alpha \beta}\left|\dot{\gamma}^{\beta}\right|, h^{\alpha \beta}=q^{\alpha \beta} h^{(\beta)}, \\
& h^{(\beta)}=h_{0}\left(\frac{s^{s}-s^{\beta}}{s^{s}-s_{0}}\right)^{a}, s^{s}=s_{0}^{s}\left(\frac{\hat{p}}{\gamma_{s}}\right)^{\hat{m}}
\end{aligned}
$$

where, $h^{\alpha \beta}$ is the strain hardening rate in the slip system $\alpha$ shearing on the slip system $\beta, q^{\alpha \beta}$ is the hardening matrix. $h^{(\beta)}$ is defined by initial CRSS $s_{0}$, saturation strain rate value $\dot{\gamma}_{s}$ and other material-independent parameter, reference CRSS value $s_{0}^{s}$, the reference hardening rate $h_{0}$, exponential value $\hat{m}$. The parameters of the strain rate function and the strain hardening equation could be obtained in the fitting test of representative volume element (RVE).

As for the damage evolution, a simple continuum damage equation is used to couple with the finite element method [3].

$$
D=\left\{\begin{array}{cl}
0 & \left(\gamma_{m} \leq \gamma_{m, m i}\right) \\
D_{m=x}\left(\frac{\gamma_{m}-\gamma_{m, m a}}{r_{m, m x}-\gamma_{m, m i}}\right)^{n} & \left(\gamma_{\min i}<\gamma_{m}<\gamma_{m, m a x}\right) \\
D_{\max } & \left(\gamma_{m} \geq \gamma_{m, m a x}\right)
\end{array}\right.
$$

where, $\gamma_{m, i n i}$ is the initial shear strain, $D_{\max }$ the maximum value of damage factor, $\gamma_{\operatorname{m} m a x}$ is the maximum shear strain of the 12 slip systems in FCC crystal, $\mathrm{M}$ is the exponent that controls the shape of these curves. The damage initiates when $\gamma_{m}$ is higher than $\gamma_{m, i n i}$ in each integration point of finite elements. As $\gamma_{m}$ reaches the $\gamma_{\max }$ in one integration point, the damage reaches its maximum value.

\section{Figures and tables}

The conventional tensile test is modified into thermal tensile equipment. The copper electrode is used to attain the thermocouple onto aluminum alloy to control the current temperature and the tensile behavior is controlled by the computer to precisely measure the stress-strain curves. Other strain rate and temperature jump tests are also used to obtain the thermal activated energy and volume, $\Delta \mathrm{F}$ and $\Delta \mathrm{V}[9]$.

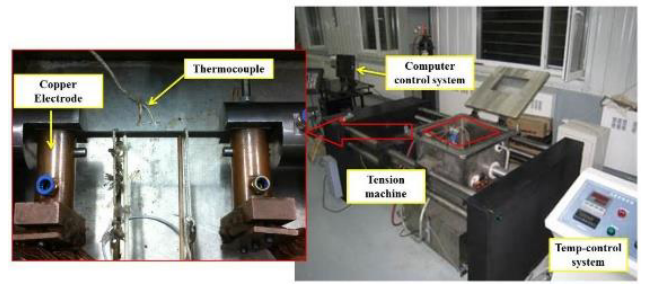

Figure 3. The detailed equipments for aluminum tensile experiment.
In this section, the RVE simulation test is used to obtain the 12 material parameters $\rho_{\mathrm{m}}, v_{\mathrm{id}}, \Delta \mathrm{F}, \Delta \mathrm{V}, q, h_{0}$, $a, s_{0}^{z}, \widehat{m}(\theta=300 K), \widehat{m}(\theta=373 K), \hat{m}(\theta=473 K)$, $\hat{m}(\theta=573 \mathrm{~K})$ and to verify the experimental parameters acquired from the jump tests. The cube size is about $0.3 \mathrm{~mm} \times 0.3 \mathrm{~mm} \times 0.3 \mathrm{~mm}$, which contains 50 crystallographic orientations, representing an aluminium aggregate. The Voronoi tessellation is used to divide the cube, and the morphology of each single crystal is similar to the real grain morphology. 5659 tetrahedron elements are conducted to mesh the polycrystalline to better suit the element shape and obtain a better grain mesh. The mechanical fitting test is revealed in the stress-strain curves and a good agreement is acquired to simulate the material parameters, which can be further used in the practical simulation of realistic tensile behavior in Section 4.
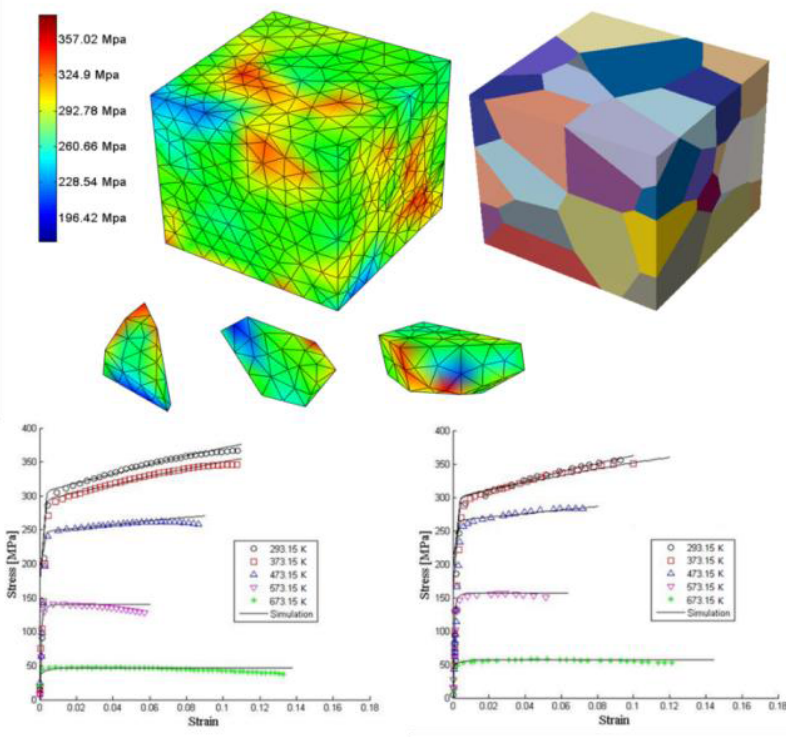

Figure 4. Schematic illustration of parameters determination of RVE finite element simulation.

\section{Thermal tensile simulation}

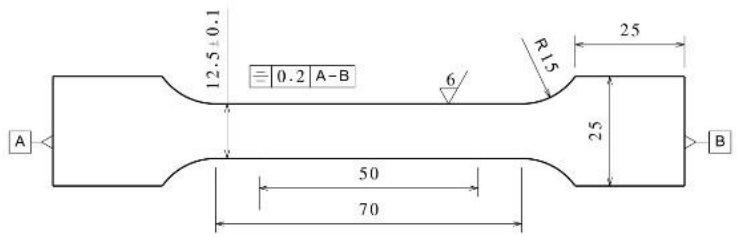

Figure 5. The dimension parameter of specimen for tensile test.

To verify this multiscale framework of CPFEM method, an actual tensile test is compared with the simulation results on 5052 aluminum alloy.

Moreover, a Taylor-based homogenization model is also implemented with the crystal plasticity model to compare the mechanical results. The material parameters are obtained using RVE simulation. Here, as shown in Fig. 5, the CPFEM simulation results agrees well with the experimental data and also the Taylor model at all the three elevated temperatures. In both the simulation and 
experiment, as the temperature gets higher, the strain hardening phenomenon is numerically weakened by the thermal affected strain hardening equation. The damage point of each temperature varies because of the damage model.

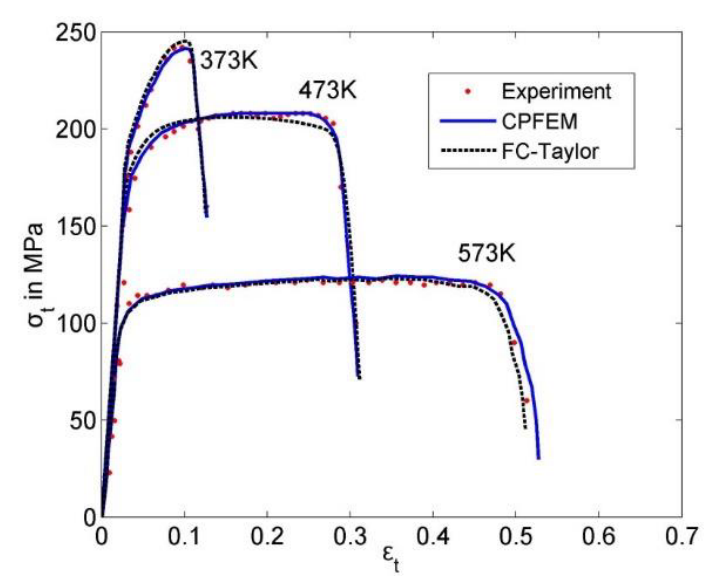

Figure 6. Comparison between experimental data and crystal plasticity methods at various temperatures.

The stress concentration is shown in Fig. 6 according to the orientation mismatch among adjacent grains, which is originally caused by the single crystal anisotropy. Generally isotropic $\mathrm{J} 2$ plasticity cannot reveal this kind of concentration. The damage evolution process is simulated in the ABAQUS, and the subroutine UMAT is implemented.

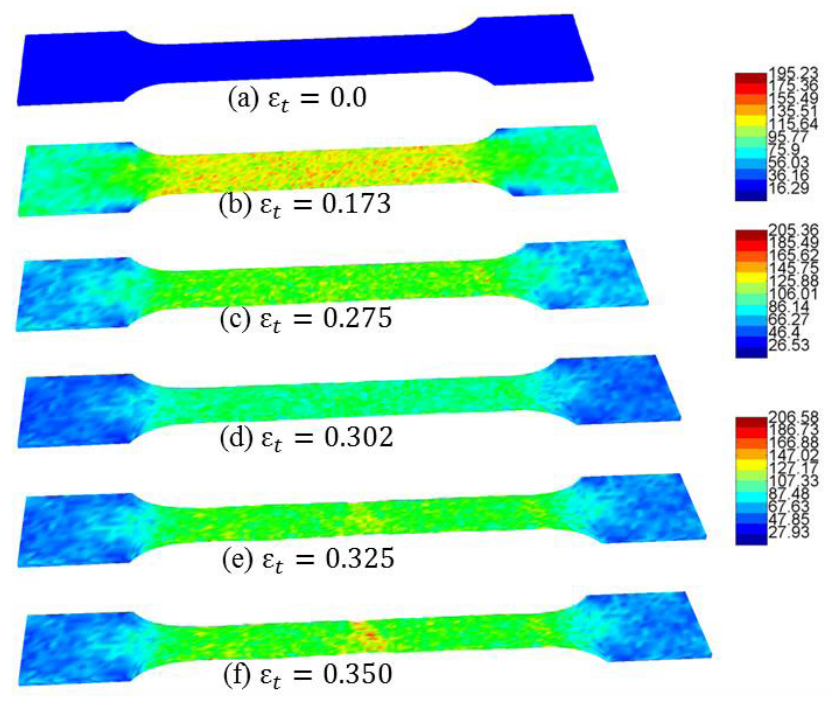

Figure 7. Von Mises stress distribution (maximum shear strain damage model: $\theta=473 \mathrm{~K}, \gamma_{\operatorname{mini}}=0.223, \gamma_{\max }=0.24 \mathrm{C}$, $\mathrm{D}_{\max }=0.8, \mathrm{M}=3.0$ ).

As shown in Fig. 7, the damage evolution is revealed as the logarithmic strain exceeds a certain value. The most significant phenomenon occurs as the $\varepsilon_{t}=0.325$, when all the elements of the middle section has entered into the damage level. The damage elements increases as the strain is enhanced and it is similar to the void evolution in the material behavior of aluminum alloy.

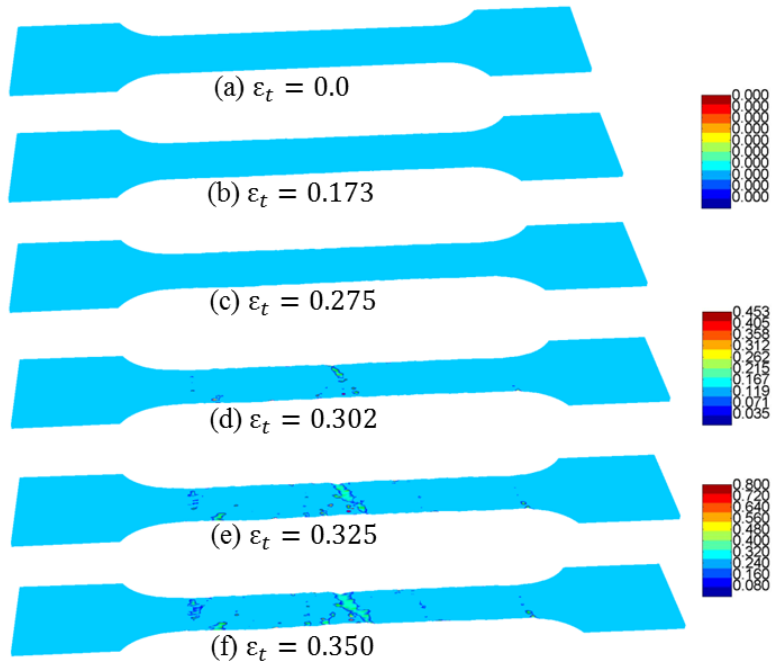

Figure 8. Damage factor distribution (maximum shear strain damage model: $\theta=473 \mathrm{~K}, \gamma_{\operatorname{mini}}=0.223, \gamma_{\max }=0.249$, $\left.\mathrm{D}_{\max }=0.8, \mathrm{M}=3,0\right)$.

\section{Conclusion and discussion}

A crystal plasticity extended finite element method is implemented in a multiscale framework, and it could be used to consider the thermal effects and the damage evolution. The local damage evolution is shown in the simulation of commercial software ABAQUS, and the mechanical response is calculated using the subroutine UMAT. The crystal plasticity model is revised by the thermal activated mechanism and a RVE simulation is able to obtain all the material parameters. Therefore, any FCC-structure material behavior could be calculated according to the fitting test of RVE simulation. The mechanical response agrees well with the experimental data.

The stress concentration is shown in Fig. 6 according to the orientation mismatch among adjacent grains, which is originally caused by the single crystal anisotropy. Generally isotropic J2 plasticity cannot reveal this kind of concentration. The damage evolution process is simulated in the ABAQUS, and the subroutine UMAT is implemented.

In our future study, different crystal structures are considered to develop the subroutine and other polycrystalline behaviors are the goals of our research, such as the grain boundary property, the recrystallization behavior. The temperature field is also a significant problem to be solved coupled with the mechanical field in the finite element analysis.

\section{Acknowledgements}

This work was funded by the Key Project of the National Natural Science Foundation of China (No.11272075), China Postdoctoral Science Foundation (2014M561223) and the Fundamental Research Funds for the Central Universities (DUT14RC(3)032). 


\section{References}

1. B. Peeters, S.R. Kalidindi, P. Van Houtte and E. Aernoudt, Acta Mater. 48, 2123-2133 (2000)

2. C.C., Tasan, J.P.M. Hoefnagels, M. Diehl, D. Yan, F. Roters and D. Raabe, Int. J. Plasticity 63, 198-210 (2014)

3. J. Kim and J.W. Yoon, Int. J. Plasticity 73, 3-23 (2015)

4. L. Chen, J. Chen, R.A. Lebensohn, Y.Z. Ji, T.W. Heo, S. Bhattacharyya, K. Chang, S. Mathaudhu, Z.K. Liu and L.Q. Chen, Comput. Method Appl. M. 285, 829-848 (2015)

5. D. Raabe and F. Roters, Int. J. Plasticity 20, 339-361 (2004)

6. J.R. Mayeur, I.J. Beyerlein, C.A. Bronkhorst and H.M. Mourad, Int. J. Plasticity 65, 206-225 (2015)

7. S. Keshavarz and S. Ghosh, Acta Mater 61, 65496561 (2013)

8. G.B. Sarma and B. Radhakrishnan, Mater. Sci. Eng., A 385, 91-104 (2004)

9. D. Caillard and J.L. Martin, Thermal activated mechanisms in crystal plasticity (ELSEVIER Ltd, Oxford, 2003) 